\title{
On Computing Normalized Coprime Factorizations of Rational Matrices
}

\author{
A. Varga \\ DLR - Oberpfaffenhofen \\ Institute of Robotics and System Dynamics \\ D-82234 Wessling, F.R.G. \\ E-mail: Andreas.Varga@dlr.de
}

\begin{abstract}
We propose a new computational approach based on descriptor state space algorithms to compute normalized coprime factorizations of rational matrices.
\end{abstract}

\section{Introduction}

Let $G=(E, A, B, C, D)$ be a minimal order linear timeinvariant continuous- or discrete-time regular descriptor system, denoted also $G=\left[\begin{array}{c|c}A-\lambda E & B \\ \hline C & D\end{array}\right]$ with the rational transfer function matrix (TFM) $G(\lambda)=$ $C(\lambda E-A)^{-1} B+D$, where $\lambda=s$ or $\lambda=z$, in accordance with the type of the system. We denote with $G^{\sim}$ the conjugate TFM, where $G^{\sim}(s)=G^{T}(-s)$ in continuoustime and $G^{\sim}(z)=G^{T}(1 / z)$ in discrete-time. A right coprime factorization (RCF) $G=N M^{-1}$ with $N$ and $M$ coprime stable rational matrices satisfying the additional condition $M^{\sim} M+N^{\sim} N=I$, is called a normalized right coprime factorization (NRCF).

The computation of NRCF of non-proper TFMs has been considered for continuous-time systems in [1]. The proposed procedure relies on a particular descriptor representation with $E=\operatorname{diag}(I, 0)$ and $D=0$ and involves the solution of two standard Riccati equations. Since the initial reduction can lead to unnecessary accuracy loss, this approach raises serious concerns from numerical point of view. For discrete-time systems, apparently there are no results for non-proper TFMs.

In this paper we propose a completely general method to compute NRCFs of arbitrary continuous- or discretetime TFMs. The procedure given below can be seen as a constructive proof of the following main result.

Theorem. An arbitrary rational matrix $G(\lambda)$ can be always represented as a NRCF $G=N M^{-1}$, where $N$ and $M$ are proper rational matrices.

NRCF Procedure.

1. Compute a RCF $G=N_{1} M_{1}^{-1}$ such that both $N_{1}$ and $M_{1}$ are proper TFMs.

2. Solve the spectral factorization problem $M_{1}^{\sim} M_{1}+$ $N_{1}^{\sim} N_{1}=G_{o}^{\sim} G_{o}$.

3. Compute $M=M_{1} G_{o}^{-1}$ and $N=N_{1} G_{o}^{-1}$.
Step 1 reduces essentially the NRCF problem to one for a proper TFM. This step can be performed by using an algorithm proposed in [2]. The NRCF is computed at steps 2 and 3 by solving a spectral factorization problem for a proper system in a descriptor representation with nonsingular $E$. This can be done by solving appropriate descriptor Riccati equations arising from the descriptor variants of standard spectral factorization algorithms. From numerical point of view, the proposed approach represents a completely satisfactory computational solution to determine NRCFs. Applied to $G^{T}$, it can be equally used to compute normalized left coprime factorizations too.

\section{Proper rational factorization}

In this section we consider the problem at step 1 of the NRCF Procedure to compute a RCF $G=N_{1} M_{1}^{-1}$ such that $N_{1}$ and $M_{1}$ are proper. For a given minimal descriptor represention $G=(E, A, B, C, D)$, a general algorithm for this computation has been proposed in [2]. Here we need only the first part of this algorithm, which determines a state feedback matrix $F$ to eliminate the impulsive behavior of the system. With the determined $F$, the proper factors result as [3]:

$$
\left[\begin{array}{l}
N_{1} \\
M_{1}
\end{array}\right]=\left[\begin{array}{c|c}
A+B F-\lambda E & B \\
\hline C+D F & D \\
F & I
\end{array}\right] .
$$

By applying an appropriate orthogonal similarity transformation, the given system matrices can be put in a SVD-like coordinate form

$$
G=\left[\begin{array}{cc|c}
A_{11}-\lambda E_{11} & A_{12} & B_{1} \\
A_{21} & A_{22} & B_{2} \\
\hline C_{1} & C_{2} & D
\end{array}\right],
$$

where $E_{11} \in \mathbb{R}^{r \times r}$ is nonsingular. Because of minimality assumption, rank $B_{2}=n-r$ and we can take $F=\left[\begin{array}{ll}0 & F_{2}\end{array}\right]$, where $F_{2}$ is chosen such that the matrix $A_{22}+B_{2} F_{2}$ is non-singular and well-conditioned. By this choice the pair $(E, A+B F)$ is regular and has $r$ finite eigenvalues and $n-r$ simple eigenvalues at infinity. 
The resulting factors, obtained after eliminating the non-dynamic modes with the help of well-known residualization formulas, are $\left[\begin{array}{l}N_{1} \\ M_{1}\end{array}\right]=(\bar{E}, \bar{A}, \bar{B}, \bar{C}, \bar{D})$, where $\bar{A}=A_{11}-\left(A_{12}+B_{1} F_{2}\right)\left(A_{22}+B_{2} F_{2}\right)^{-1} A_{21}$, $\bar{E}=E_{11}, \bar{B}=B_{1}-\left(A_{12}+B_{1} F_{2}\right)\left(A_{22}+B_{2} F_{2}\right)^{-1} B_{2}$,

$$
\begin{aligned}
& \bar{C}=\left[\begin{array}{l}
\bar{C}_{1} \\
\bar{C}_{2}
\end{array}\right]=\left[\begin{array}{l}
C_{1}-\left(C_{2}+D F_{2}\right)\left(A_{22}+B_{2} F_{2}\right)^{-1} A_{21} \\
-F_{2}\left(A_{22}+B_{2} F_{2}\right)^{-1} A_{21}
\end{array}\right] \\
& \bar{D}=\left[\begin{array}{l}
\bar{D}_{1} \\
\bar{D}_{2}
\end{array}\right]=\left[\begin{array}{l}
D-\left(C_{2}+D F_{2}\right)\left(A_{22}+B_{2} F_{2}\right)^{-1} B_{2} \\
I-F_{2}\left(A_{22}+B_{2} F_{2}\right)^{-1} B_{2}
\end{array}\right]
\end{aligned}
$$

For a proper system $F_{2}=0$ and if $E$ is nonsingular then we can simply take $N_{1}=G$ and $M_{1}=I$.

\section{The computation of NRCF}

Starting with the proper factorization $G=N_{1} M_{1}^{-1}$ computed above, we derive the formulas to compute the NRCF to perform the steps 2 and 3 of the NRCF Procedure. If the original system is proper, then the following result is a straightforward extension of [4] and [5] for descriptor representations.

Proposition 1. Let $\bar{X}$ be the symmetric stabilizing solution of the generalized continuous-time algebraic Riccati equation (GCARE)

$$
\begin{aligned}
0= & \bar{E}^{T} \bar{X} \bar{A}+\bar{A}^{T} \bar{X} \bar{E}+\bar{C}^{T} \bar{C} \\
& -\left(\bar{E}^{T} \bar{X} \bar{B}+\bar{C}^{T} \bar{D}\right) \bar{R}^{-1}\left(\bar{B}^{T} \bar{X} \bar{E}+\bar{D}^{T} \bar{C}\right),
\end{aligned}
$$

where $\bar{R}=\bar{D}^{T} \bar{D}$, or of the generalized discrete-time algebraic Riccati equation (GDARE)

$$
\begin{aligned}
& \bar{E}^{T} \bar{X} \bar{E}=\bar{A}^{T} \bar{X} \bar{A}+\bar{C}^{T} \bar{C} \\
& \quad-\left(\bar{A}^{T} \bar{X} \bar{B}+\bar{C}^{T} \bar{D}\right) \bar{R}^{-1}\left(\bar{B}^{T} \bar{X} \bar{A}+\bar{D}^{T} \bar{C}\right),
\end{aligned}
$$

where $\bar{R}=\bar{D}^{T} \bar{D}+\bar{B}^{T} \bar{X} \bar{B}$. Let $\bar{F}$ be the corresponding stabilizing feedback matrix computed in continuous-time as

$$
\bar{F}=-\bar{R}^{-1}\left(\bar{B}^{T} \bar{X} \bar{E}+\bar{D}^{T} \bar{C}\right)
$$

or in discrete-time as

$$
\bar{F}=-\bar{R}^{-1}\left(\bar{B}^{T} \bar{X} \bar{A}+\bar{D}^{T} \bar{C}\right) .
$$

Then $G_{o}=(\bar{E}, \bar{A}, \bar{B},-\overline{H F}, \bar{H})$ with $\bar{H}^{T} \bar{H}=\bar{R}$, satisfies $N_{1}^{\sim} N_{1}+M_{1}^{\sim} M_{1}=G_{o}^{\sim} G_{o}$. The factors $M=G_{o}^{-1}$ and $N=N_{1} G_{o}^{-1}$ of the NRCF can be expressed as

$$
\left[\begin{array}{c}
N \\
M
\end{array}\right]=\left[\begin{array}{c|c}
\bar{A}+\bar{B} \bar{F}-\lambda \bar{E} & \bar{B} \bar{H}^{-1} \\
\hline \bar{C}_{1}+\bar{D}_{1} \bar{F} & \bar{D}_{1} \bar{H}^{-1} \\
\bar{F} & \bar{H}^{-1}
\end{array}\right] .
$$

For the numerical solution of GCARE and GDARE, consider the extended Hamiltonian pencil (EHP)

$$
L-\lambda P:=\left[\begin{array}{ccc}
\bar{A}-\lambda \bar{E} & 0 & \bar{B} \\
-\bar{C}^{T} \bar{C} & -\bar{A}^{T}-\lambda \bar{E}^{T} & -\bar{C}^{T} \bar{D} \\
\bar{D}^{T} \bar{C} & \bar{B}^{T} & \bar{D}^{T} \bar{D}
\end{array}\right]
$$

and, respectively, the extended simplectic pencil (ESP)

$$
L-\lambda P:=\left[\begin{array}{ccc}
\bar{A}-\lambda \bar{E} & 0 & \bar{B} \\
-\bar{C}^{T} \bar{C} & \bar{E}^{T}-\lambda \bar{A}^{T} & -\bar{C}^{T} \bar{D} \\
\bar{D}^{T} \bar{C} & \lambda \bar{B}^{T} & \bar{D}^{T} \bar{D}
\end{array}\right] .
$$

The following result allow to compute the solution of GCARE and GDARE by solving an appropriate generalized eigenvalue problems $[6,7]$.

Proposition 2. Let $V$ be a basis matrix for the maximal dimension stable deflating subspace $\mathcal{V}$ of the EHP (5) or ESP (6) and let $V=\left[\begin{array}{lll}V_{1}^{T} & V_{2}^{T} & V_{3}^{T}\end{array}\right]^{T}$ be partitioned in accordance with $L$ and $P$ in (5) or (6). Then $V_{1} \in \mathbb{R}^{n \times n}$ is nonsingular, and the stabilizing solution $\bar{X}$ of the GCARE (1) or GDARE (2), and the corresponding stabilizing feedback $\bar{F}$ in (3) and (4) respectively, can be computed as

$$
\bar{X}=V_{2}\left(\bar{E} V_{1}\right)^{-1}, \quad \bar{F}=V_{3} V_{1}^{-1} .
$$

Both the EHP and ESP being regular, standard numerical techniques can be employed to compute $V$ [8].

\section{References}

[1] P. M. M. Bongers and O. H. Bosgra. Normalized coprime factorizations for systems in generalized statespace form. IEEE Trans. Autom. Control, 38:348-350, 1993.

[2] A. Varga. Generalized Schur methods to compute coprime factorizations of rational matrices. In Proc. 1st Asian Control Conference, Tokyo, Japan, vol. 3, pp. 89-92, 1994.

[3] F. Y. Wang and M. J. Balas. Doubly coprime fractional representations of generalized dynamical systems. IEEE Trans. Autom. Control, AC-34:733-744, 1989.

[4] M. Vidyasagar. Normalized coprime factorization for nonstrictly proper systems. IEEE Trans. Autom. Control, 33:300-301, 1988.

[5] P. M. M. Bongers and P. S. C. Heuberger. Discrete normalized coprime factorization. In A. Bensoussan and J. L. Lions, editors, Proc. 9th INRIA Conf. Analysis and Optimization of Systems, vol. 144, Lect. Notes Control and Inf. Scie., pp. 307-313, SpringerVerlag, Berlin, 1990.

[6] W. F. Arnold III and A. J. Laub. Generalized eigenproblem algorithms and software for algebraic Riccati equations. Proc. of IEEE, 72:1746-1754, 1984.

[7] V. L. Mehrmann. The Autonomous Linear Quadratic Control Problem, vol. 163, Lect. Notes Contr. Inf. Scie., Springer Verlag, Berlin, 1991.

[8] P. Van Dooren. The generalized eigenstructure problem in linear systems theory. IEEE Trans. Autom. Control, 26:111-129, 1981. 